\title{
Design of a Dynamic Ship Draft Detection System in Inland Navigation
}

\author{
Jiahai Zhou', a, Panpan Zhang 1, 2, b \\ ${ }^{1}$ China Waterborne Transport Research Institute, Beijing, 100088, China; \\ ${ }^{2}$ Key Laboratory of Logistics Equipment \& Control Engineering, Beijing, 100088, China. \\ azhoujh@wti.ac.cn, bzhangpanpan@wti.ac.cn
}

\begin{abstract}
With the development of China's economic development, the waterborne transportation plays more and more important role. However, ultra-draft issues in inland shipping are becoming a big problem which will bring unsafe factors and affect the efficiency of inland navigation. Thus, ship waterline inspection are compulsive. In this paper, the draft detection techniques were summarized and the sensor array technique was chosen to set up the detection platform. Besides, a kind of ship draft detection equipment was designed and characteristics of the detection system were analyzed.
\end{abstract}

Keywords: Ship draft; ultra-draft; sensor array; detection platform; flexible adjustment.

\section{Introduction}

The waterborne transportation which has advantages of low cost and large volume plays more and more important part with the development of China's economic development. As the western development is carried out, the safety and efficiency of inland navigation, especially in Yangtze River area, is of great importance. However, the number of shipping accidents has been increasing, most of which were caused by exceeding the maximum draught shown in figure 1 . The accident significantly affects the safety and efficiency of the waterway [1].
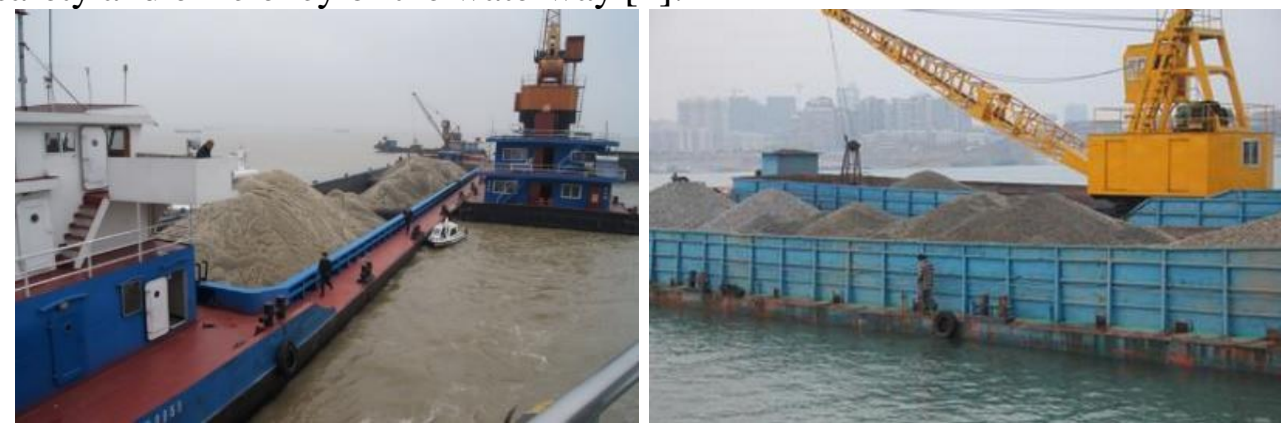

Figure 1. The phenomenon of ship overload

Draft detection now is undertaken by maritime law enforcement officers through visual inspection. Visual inspection of ship draft is direct but inefficient. In recent years, researchers have been working on the draft detection techniques to improve the waterline inspection of ships. In this paper, the draft detection techniques were summarized and a kind of ship draft detection equipment was designed. Among the waterline inspection, the sensor array method was chosen to adapt to the detection platform.

\section{Comparisons among ship draft detection methods}

There are several techniques in the area of ship draft detection. Each method has its own advantages and disadvantages.

Image recognition is a simple and low-cost way to draft detection [2]. First, a camera is used to take a picture of the ship water gauge. Then the picture is processed via the computer and the ship draft can obtained. This reliability of the image recognition method mostly relay on the accuracy of water gauge and image definition. Besides, the recognition of waterline is still a problem to be resolved. When the position of vessels leans incline, the deviation of draft detection is unacceptable.

The laser technology, wide-range laser scanning technology, array-type weak light receiving technology, and weak signal processing technology are combined into the laser screen method [3]. 
When the ship goes across the screen, the blocked section of the ship can be detected. With the help of the channel water lever gauge, the ship draft can be calculated. The advantages of this method can achieve rapid detection rate and high accuracy. The accuracy of the method is greatly affected by the turbidity degree of water and the amount of ships. It is because anti-interference ability of laser is not strong enough. Besides, the laser could do harm to the aquatic organisms.

Multi beam ultrasonic measurement is based on the multi beam forming technique. Transducers fixed underwater are used for launching the sector-shape ultrasonic beams and receiving the ultrasonic beams. According to the acoustic wave propagation characteristics, the water depth can be calculated and the ship draft can be gained. The number of the multi ultrasonic beam and the limitation of reflection angle have great influence on the detection results shown in Figure 2[4]. The procedure of the multi beam ultrasonic technology was shown in Figure 3.

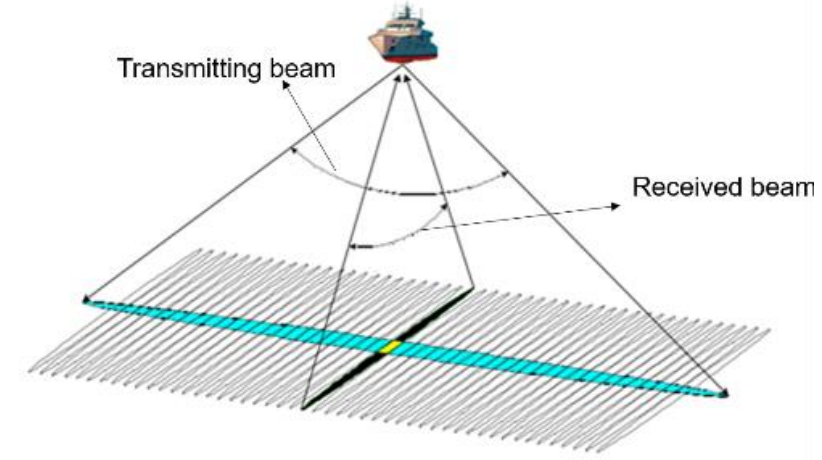

Figure 2. The schematic chart of multi beam ultrasonic measurement

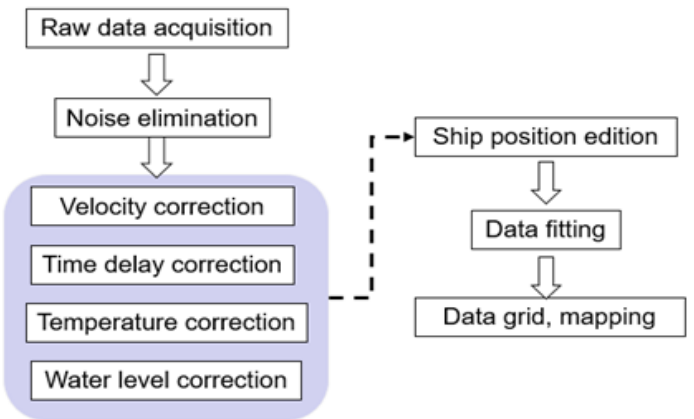

Figure 3. The procedure of multi beam ultrasonic measurement

Side scan sonar technology [5] can directly obtain the underwater patterns and it is widely used in the underwater surveying and mapping areas. But in the fields of ship draft detection, this method is not a good option because sonar is sensitive to the rough surface. And the surface of the ship is usually smooth. This method can provide a picture of the ship instead of the draft which limits the application.

Single beam array ultrasonic measurement shown in Figure 4 is derived from the multi beam ultrasonic method [6]. In this method, a number of single beam ultrasonic sensors are placed into an array. The sensor launches single ultrasonic wave and receives the reflection wave. When the vessel goes across the sensor array, ultrasonic wave can be reflected by the bottom of the vessel. And then the information of the ship bottom can be calculated in order to get the ship draft.

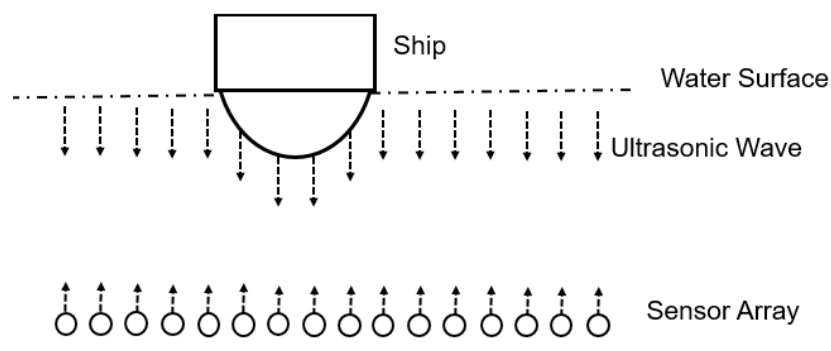

Figure 4 The diagram of single beam ultrasonic measurement 


\section{Structure of the ship draft detection system}

The ship draft detection system shown in Figure 5 is composed of data acquisition system, detection platform and data processing system. In the data acquisition system, single beam ultrasonic sensor array which is installed in a detection platform is used to get the information of the crossing ships. A detection platform was designed to facilitate the different level of water. And in extreme weather condition, the detection platform can be rotated along the river bank. The data getting from the ultrasonic sensor array were sent to the data processing system. After data were processed, the real-time ship draft can be obtained. Based on comprehensive comparisons with the detection method mentioned above, Single bean ultrasonic measurement was chosen to carry out the ship real-time draft detection.

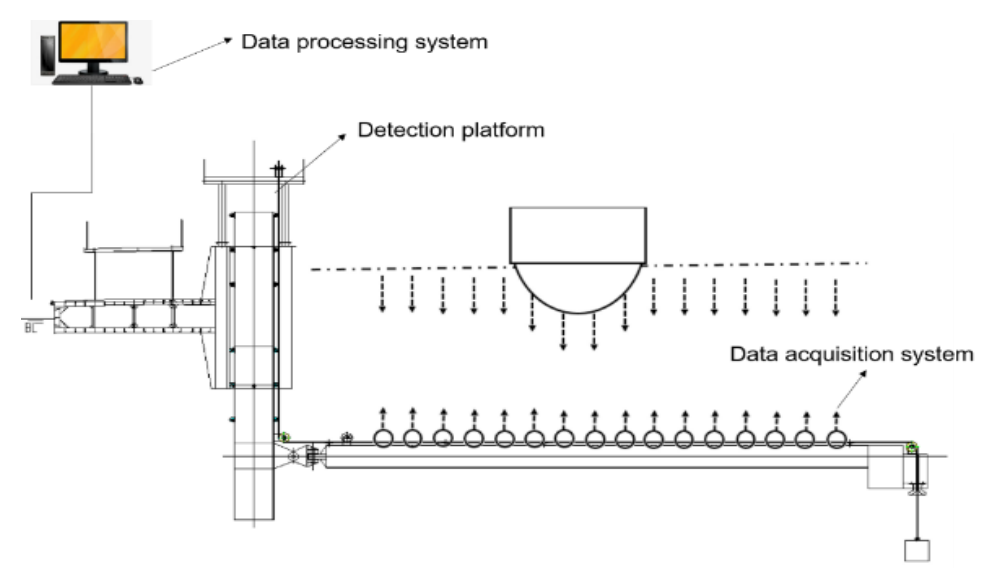

Figure 5. The diagram of the ship draft detection system

\section{Design of the draft detection platform}

In order to realize the single beam ultrasonic measure, a detection platform was designed to support the sensor array. At the same time, the detection platform has the ability of flexible adjustment for application of different working condition.

The platform shown in Figure 6 is consisted of a pontoon, a support structure, an elevating structure, an anchor block, a hoisting mechanism and an anti-swing mechanism. The hoisting mechanism was installed in the pontoon. The support structure is used for the sensor array installation. The hoisting mechanism was designed to apply for different water level. When a certain water is given, the anchor block is adopted to stabilize the platform. Besides, an anti-swing mechanism was put into use to keep the balance condition of the support structure in the flow of water.

By using the elevating structure and the hoisting mechanism, the draft detection system can work in different level of water. And in the extreme weather condition, the support structure can be adjusted to lay along the riverside.

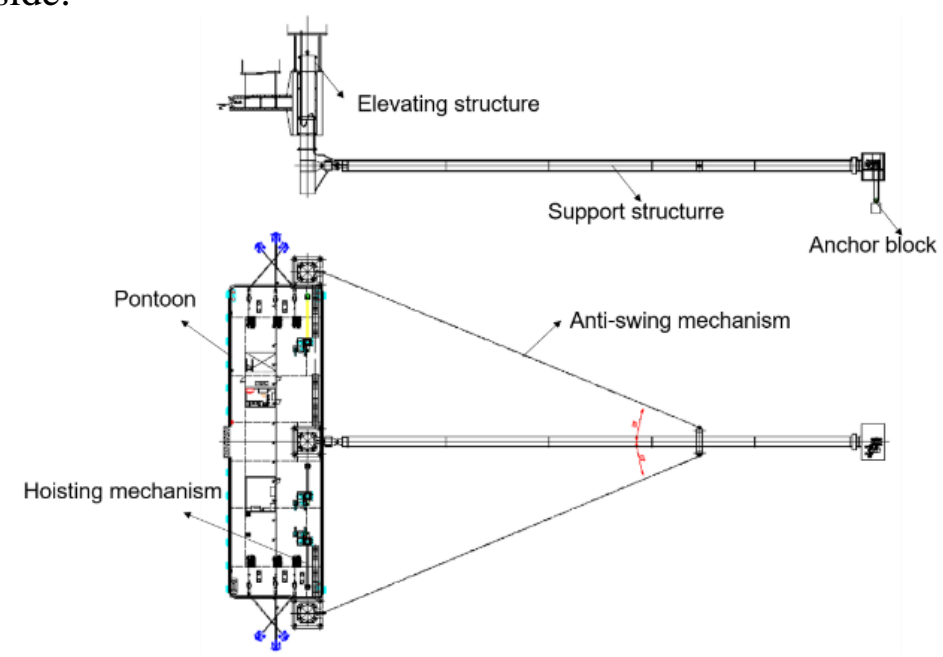

Figure 6. The diagram of the ship draft detection platform 


\section{Summary}

Ultra-draft of ships has brought unsafe issues to the inland navigation. There are different methods to detect the ship draft. Both advantages and disadvantaged were analyzed in this paper. A ship draft detection system was devised and a detection platform was designed to facilitate the ship draft inspection. This platform can be compliant with different working condition. And in future, the detection system will be manufactured and put into use in the inland navigation inspection.

\section{References}

[1] Fei Tong. Wen Zeng, Research on the Navigation Management of Ultra-draft, China Water Transport, 2008.7:32-33.

[2] Guangcheng Zhou. Application of Image Processing Technology in the Dynamic Ship Draft Detection, Nanjing University of Science \& Technology, 2006.

[3] Siyin Zhu. Research on Key Technologies of the Real-time Detection of Dynamic Ship Draft and Data Processing, Dalian Maritime University, 2011.

[4] Ulrich Lenk. Multibeam Data Processing: a Detailed Algorithm to Compute (Adaptive) Triangulation and Potential Indications of Data Quality. The Hydrographical Journal. 2003, (107): 3-10.

[5] Farr H K. Multi beam bathymetric Sonar: Sea Beam and Hydro hart. Manual Geodesy. 1980. 4(2): 77 9.

[6] Hua Su. Study on Dynamic Measurement Method of Ship Draught, Wuhan University of Technology, 2008. 